\title{
In vitro Gene Expression Assay
}

National Cancer Institute

\section{Source}

National Cancer Institute. In vitro Gene Expression Assay. NCI Thesaurus. Code C158251.

A method that detects gene transcription associated with an in vitro cellular stimulus, through detection of the resulting transcribed mRNA or translated protein. 